University of South Carolina

Scholar Commons

$11-15-2005$

\title{
Photovoltaic Effect in Bent Quantum Wires in the Ballistic Transport Regime
}

Yuriy V. Pershin Dr

University of South Carolina - Columbia, pershin@physics.sc.edu

Carlo Piermarocchi

Follow this and additional works at: https://scholarcommons.sc.edu/phys_facpub

Part of the Physics Commons

Publication Info

Published in Physical Review B, ed. Gene D. Sprouse, Volume 72, Issue 19, 2005, pages

195340-1-195340-4.

Pershin, Y. V., \& Piermarocchi, C. (2005). Photovoltaic effect in bent quantum wires in the ballistic transport regime. Physical Review B, 72(19), 195340-1 - 195340-4. DOI: 10.1103/PhysRevB.72.195340 (c) Physical Review B, 2005, American Physical Society

This Article is brought to you by the Physics and Astronomy, Department of at Scholar Commons. It has been accepted for inclusion in Faculty Publications by an authorized administrator of Scholar Commons. For more information, please contact digres@mailbox.sc.edu. 


\title{
Photovoltaic effect in bent quantum wires in the ballistic transport regime
}

\author{
Yuriy V. Pershin* and Carlo Piermarocchi \\ Department of Physics and Astronomy, Michigan State University, East Lansing, Michigan 48824-2320, USA
}

(Received 28 July 2005; revised manuscript received 6 September 2005; published 29 November 2005)

\begin{abstract}
A scheme for the generation of a photocurrent in bent quantum wires is proposed. We calculate the current using a generalized Landauer-Büttiker approach that takes into account the electromagnetic radiation. For circularly polarized light, it is demonstrated that the curvature in the bent wire induces an asymmetry in the scattering coefficients for left and right moving electrons. This asymmetry results in a current at zero bias voltage. The effect is due to the geometry of the wire which transforms the photon angular momentum into translational motion for the electrons. Possible experimental realizations of this scheme are discussed.
\end{abstract}

DOI: 10.1103/PhysRevB.72.195340

Recently many schemes for photocurrent generation in confined electron systems have been investigated. ${ }^{1-18}$ In particular, mechanisms of photocurrent generation by circularly polarized radiation have been considered in quantum rings ${ }^{15,17}$ and helical quantum wires. ${ }^{16}$ These geometries are particularly interesting because they can transform angular momentum (from the photon circular polarization) to translational motion (electron current). ${ }^{15}$ The experimental realization of these schemes has not yet been reported. In fact, the detection of the photocurrent in isolated quantum rings is experimentally challenging and helical quantum wires cannot be fabricated using standard growth techniques.

In this paper, we consider the photocurrent induced in ballistic quantum wires bent as in Fig. 1. Quantum wires of this geometry can be easily fabricated using standard semiconductor growth techniques, like, for instance, $V$ grooving. ${ }^{19}$ The setup in Fig. 1 can also be realized by bending a single carbon nanotube on a surface. It is known that a photocurrent can be generated in straight nanotubes by superposition of single-photon and two-photon transitions. ${ }^{20}$ The effect we discuss here is due to single-photon transitions and relies only on the particular curved geometry of the wire. Using a generalized Landauer-Büttiker approach based on a scattering theory, we show that the photocurrent can be strong in this geometry. In a GaAs based quantum wire under a radiation of $33 \mathrm{~mW} / \mathrm{cm}^{2}$, we obtain a current of the order of $10 \mathrm{pA}$, which is measurable with standard methods. The circularly polarized radiation propagating perpendicularly to the wire plane induces on the electrons in the curved region a sliding potential of the form $V(s / R \pm \omega t)$, where $s$ is the position along the wire, $R$ is the radius of curvature, and $\hbar \omega$ is the radiation energy. This sliding potential is an asymmetric scattering potential for left and right moving electrons, and the difference in the transmission probabilities results in a steady current. The classical interpretation of the effect is that only the electrons moving in the same direction of the sliding potential are accelerated. Moreover, we found that quantum interference plays an important role in the current. In fact, the energy dependence of the current shows not only a peak at the Fermi energy, but also several additional peaks. These additional peaks are due to the quantum interference of transmitted and reflected waves at the points where the curvature of the wire changes.
PACS number(s): 73.21.Hb, 72.40.+w, 72.30.+q

As shown in Fig. 1, we model the curved quantum wire in the $x-y$ plane using two straight quantum wires (regions 1 and 3 ) connected by an arc of a radius $R$ (region 2). On the opposite side the straight quantum wires are connected to the left $(L)$ and right $(R)$ electron reservoirs. The arc length is given by $L=\varphi R$, where $\varphi$ is the arc angle. We assume that a circularly polarized electromagnetic radiation propagates in the $z$ direction, perpendicular to the $x-y$ plane. Experimentally, an electromagnetic cavity can be used to confine the radiation, enhance the radiation-electron coupling, and increase the current. A radiation acting on the straight segments of the wire will not generate a current, no matter what polarization is used. We will, therefore, neglect the effect of the radiation on the straight segments. The electron motion along the curved wire is one-dimensional, and we define a parameter $s$ which indicates the position along the wire. For simplicity, let us select the point $s=0$ at the contact of regions 1 and 2 .

The single-electron Hamiltonian in the effective-mass approximation is given by

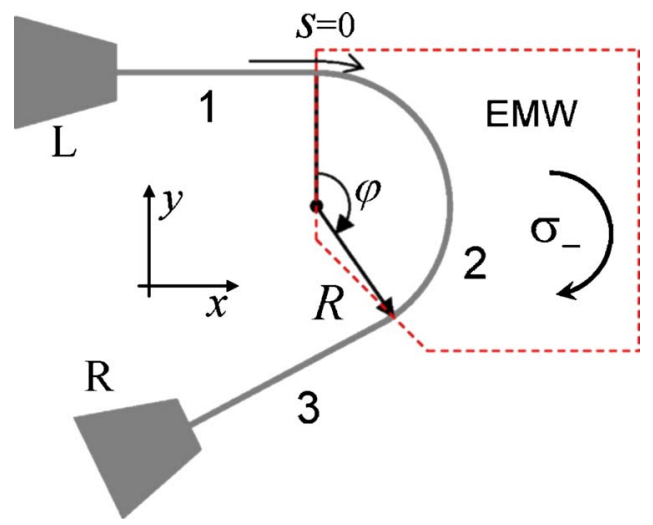

FIG. 1. (Color online) Curved quantum wire irradiated by a circularly polarized electromagnetic wave (EMW) with the electric field component precessing in $x-y$ plane. It is assumed in our model that the curved quantum wire consists of two straight segments 1 and 3 connected by an arc 2 . 


$$
H=-\frac{\hbar^{2}}{2 m^{*}} \frac{\partial^{2}}{\partial s^{2}}+(\theta(s)-\theta(s-L))\left[-\mathbf{d E}-U_{g}\right],
$$

where $m^{*}$ is the effective mass, $\theta(\ldots)$ is the step function, and $\mathbf{d}=-e \mathbf{r}$ is the dipole moment. The electric field in the radiation is written as $\mathbf{E}=E_{0} \cos (\omega t) \hat{x} \pm E_{0} \sin (\omega t) \hat{y}$, where $E_{0}$ and $\omega$ are the electric field amplitude and frequency, $\hat{x}$ and $\hat{y}$ are unit vectors in the $x$ and $y$ directions, and \pm corresponds to a $\sigma_{ \pm}$circular polarization. The first term in Eq. (1) is the kinetic energy, the second term is the dipolar interaction with the radiation, and the third term is the geometrical potential $U_{g}=\hbar^{2} /\left(8 m^{*} R^{2}\right)$, which describes the effect of the curvature. $^{24,25}$ The factor $(\theta(s)-\theta(s-L))$ makes the second and third terms different than zero only in the bent segment (region 2). Moreover, we assume that the curved quantum wire is narrow in the transverse directions, so that our model is limited only to electrons within the lowest transverse subband.

Using the substitution $y=R \cos (s / R)$ and $x=R \sin (s / R)$, we can rewrite the interaction term in Eq. (1) as

$$
-\mathbf{d E}=2 e R E_{0} \sin \left(\frac{s}{R} \pm \omega t\right) .
$$

According to Eq. (2), the electrons in the constant curvature segment are subjected to a potential that moves forward or backward depending on the helicity of the circularly polarized light. A similar sliding potential describes the interaction of electrons with a traveling acoustic wave. ${ }^{21}$ In our case, the effective wavelength of the traveling wave is determined by the quantum wire curvature and equals to $2 \pi R$.

We can write the electric current from the left $(L)$ to the right $(R)$ reservoirs using a generalization ${ }^{5,21,22}$ of the Landauer-Büttiker formula ${ }^{23}$ that takes into account the radiation

$I=\frac{2 e}{h} \sum_{n} \int_{0}^{\infty}\left[T_{R, L}(E+n \hbar \omega, E) f_{\mu_{L}}-T_{L, R}(E+n \hbar \omega, E) f_{\mu_{R}}\right] d E$.

Here $e$ is the electron charge and $T_{R, L}(E+n \hbar \omega, E)$ is the probability that an electron of energy $E$ in the left reservoir is transmitted to the right reservoir in a state of energy $E$ $+n \hbar \omega$. Since we are going to study the current in the absence of external bias, i.e., at $\mu_{L}=\mu_{R}=\mu$, Eq. (3) can be rewritten as

$$
I=\frac{2 e}{h} \int_{0}^{\infty} \Delta T(E) f_{\mu} d E,
$$

where

$$
\Delta T(E)=\sum_{n}\left[T_{R, L}(E+n \hbar \omega, E)-T_{L, R}(E+n \hbar \omega, E)\right] .
$$

We first consider the time-dependent Schrödinger equation in region 2. Taking into account only single-photon absorption and emission processes, corresponding to $n=-1,0,1$ in Eq. (5), we write the electronic wave function in the form

$$
\psi_{2}(s, t)=\sum_{n=-1}^{1} f_{n}(s) e^{-[i(E+n \hbar \omega) t] / \hbar}
$$

In what follows, we consider the case of $\sigma_{-}$polarization, as shown in Fig. 1. The $\sigma_{+}$case is analogous. Substituting (6) into the time-dependent Schrödinger equation and neglecting the terms related to multiphoton absorption and emission, we obtain

$$
\begin{gathered}
\left(E-\hbar \omega+U_{g}\right) f_{-1}+\frac{\hbar^{2}}{2 m^{*}} f_{-1}^{\prime \prime}=i e E_{0} R e^{-i(s / R)} f_{0}, \\
\left(E+U_{g}\right) f_{0}+\frac{\hbar^{2}}{2 m^{*}} f_{0}^{\prime \prime}=i e E_{0} R\left(e^{-i(s / R)} f_{1}-e^{i(s / R)} f_{-1}\right), \\
\left(E+\hbar \omega+U_{g}\right) f_{1}+\frac{\hbar^{2}}{2 m^{*}} f_{1}^{\prime \prime}=-i e E_{0} R e^{i(s / R)} f_{0} .
\end{gathered}
$$

By looking for solutions of the Eqs. (7)-(9) in the form $f_{-1}$ $=C_{-1} e^{i[\tilde{k}-(1 / R)] s}, f_{0}=C_{0} e^{i \tilde{k s}}$, and $f_{1}=C_{1} e^{i[\tilde{k}+(1 / R)] s}$, we obtain for the coefficients the system of equations

$$
\begin{gathered}
\left(E-\hbar \omega+U_{g}\right) C_{-1}-\frac{\hbar^{2}\left(\tilde{k}-\frac{1}{R}\right)^{2}}{2 m^{*}} C_{-1}-i e E_{0} R C_{0}=0, \\
\left(E+U_{g}\right) C_{0}-\frac{\hbar^{2} \widetilde{k}^{2}}{2 m^{*}} C_{0}+i e E_{0} R\left(C_{-1}-C_{1}\right)=0, \\
\left(E+\hbar \omega+U_{g}\right) C_{1}+\frac{\hbar^{2}\left(\widetilde{k}+\frac{1}{R}\right)^{2}}{2 m^{*}} C_{1}+i e E_{0} R C_{0}=0 .
\end{gathered}
$$

From the condition that the matrix in the linear system of Eqs. (10)-(12) has the determinant equal to zero, we obtain the six possible values of $\tilde{k}$. Therefore, the wave function in the second region is given by

$$
\psi_{2}(s, t)=\sum_{j=1}^{6} \sum_{n=-1}^{1} C_{n, j} e^{i\left[\tilde{k}_{j}+n(1 / R)\right] s} e^{-[i(E+n \hbar \omega) t] / \hbar},
$$

where the coefficients $C_{-1, j}$ and $C_{1, j}$ can be expressed through $C_{0, j}$ using Eqs. (10) and (12).

In order to find the transmission probability $T_{L, R}$, we solve a scattering problem selecting the wave functions in the first and third regions as

$$
\psi_{1}(s, t)=e^{i k_{0} s} e^{-[(i E t) / \hbar]}+\sum_{n=-1}^{1} r_{n} e^{-i k_{n} s} e^{-[i(E+n \hbar \omega) t] / \hbar},
$$

and

$$
\psi_{3}(s, t)=\sum_{n=-1}^{1} t_{n} e^{i k_{n} s} e^{-[i(E+n \hbar \omega) t] / \hbar}
$$

where $k_{n}=\sqrt{2 m^{*}(E+n \hbar \omega) / \hbar^{2}}$, and $r_{n}$ and $t_{n}$ are reflection and transmission coefficients, respectively. Matching the 

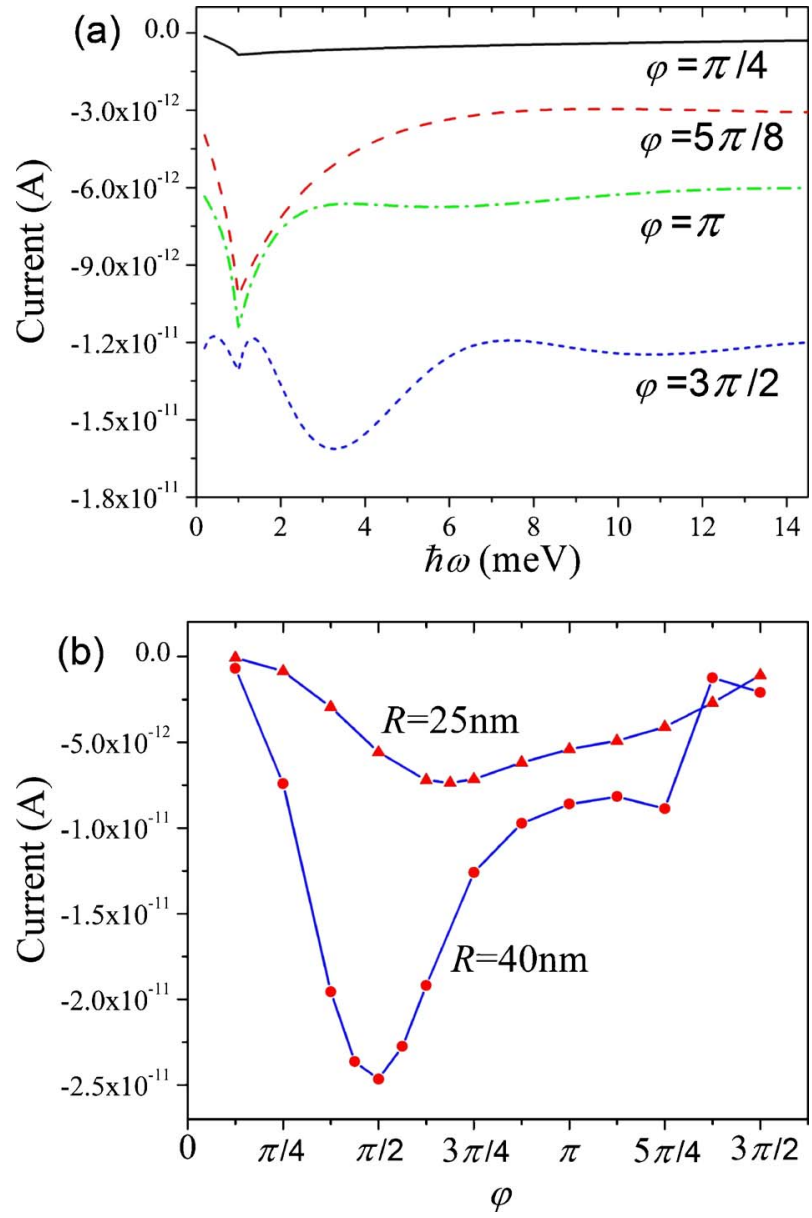

FIG. 2. (Color online) (a) A photocurrent as a function of the photon energy calculated for quantum wires having different arc lengths at fixed $R=25 \mathrm{~nm}$. (b) A photocurrent as a function of the arc length at $\hbar \omega=1 \mathrm{meV}$. These plots have been obtained using $E_{0}=500 \mathrm{~V} / \mathrm{m}, \mu=1 \mathrm{meV}, T=10 \mathrm{mK}$, and $m^{*}=0.067 m_{e}$. The electric field amplitude $E_{0}=500 \mathrm{~V} / \mathrm{m}$ corresponds to $33 \mathrm{~mW} / \mathrm{cm}^{2}$ radiation power. All the curves other than $\varphi=\pi / 4$ in (a) have been vertically shifted by steps of $0.3 \times 10^{-11} \mathrm{~A}$.

wave functions and their derivatives at the boundaries $s=0$ and $s=L$, we obtain 12 linear equations for the coefficients $r_{n}, t_{n}$, and $C_{0, j}$ with $n=-1,0,1$ and $j=1, \ldots, 6$. These equations were solved numerically, and the total transmission coefficient from the left to the right reservoir at the energy $E$ was calculated as

$$
\sum_{n=-1}^{1} T_{R, L}(E+n \hbar \omega, E)=\left|t_{0}\right|^{2}+\frac{k_{1}}{k_{0}}\left|t_{1}\right|^{2}+\theta(E-\hbar \omega) \frac{k_{-1}}{k_{0}}\left|t_{-1}\right|^{2}
$$

Using a similar scheme, we obtained the total transmission coefficient in the opposite direction and calculated the current using Eq. (4).

The results of our calculations are shown in Figs. 2 and 3. Typically, the photocurrent is negative, in agreement with the picture that the potential sliding to the right (for $\sigma_{-}$polarization) increases the transmission probability for right-moving electrons, which results in a negative current because of the
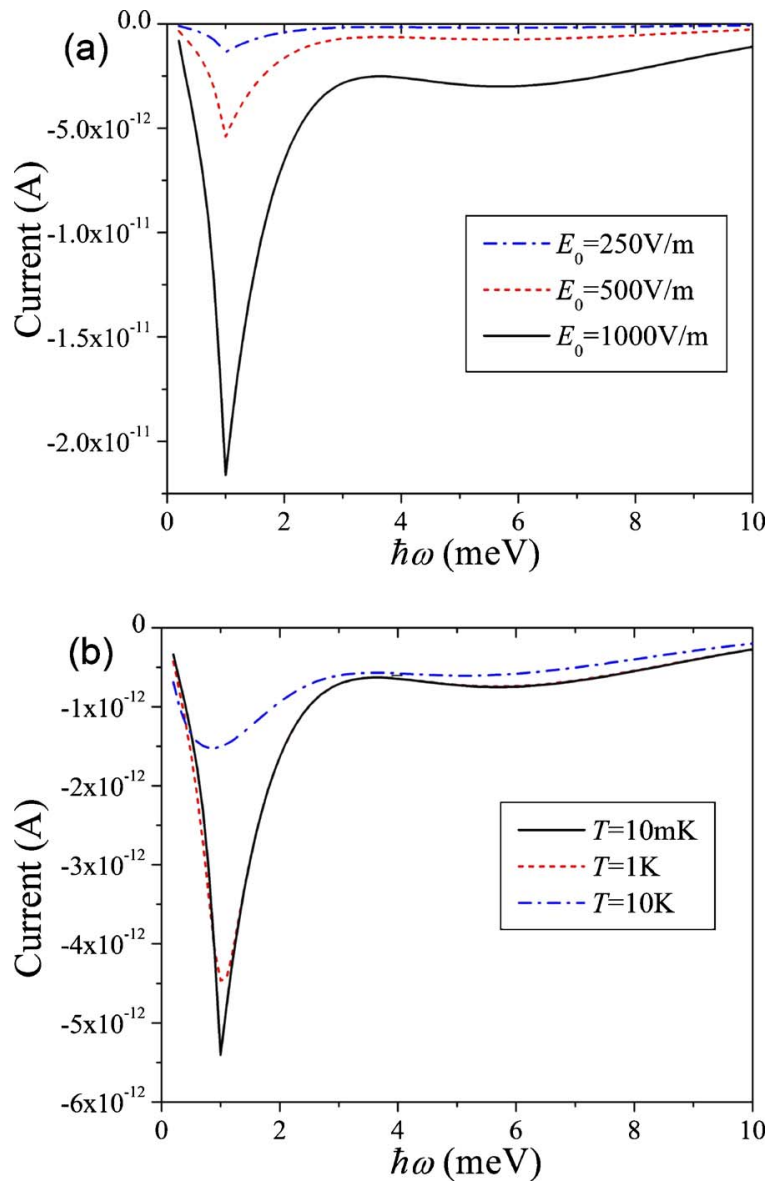

FIG. 3. (Color online) A photocurrent as a function of the photon energy calculated (a) for different radiation intensities at $T$ $=10 \mathrm{mK}$ and $(\mathrm{b})$ for different temperatures at $E_{0}=500 \mathrm{~V} / \mathrm{m}$. The other parameters values are as in Fig. 2 with $\varphi=\pi$ and $R=25 \mathrm{~nm}$.

negative electron charge $e$. However, it should be noted that the current can be positive for small values of $\hbar \omega$, as in the $\varphi=3 \pi / 2$ curve in Fig. 2(a). (Note that this curve has been shifted vertically by $1.2 \times 10^{-11} \mathrm{~A}$.) This behavior is due to quantum interference phenomena in the reflection and transmission of the electrons across the three regions. As illustrated in Fig. 2(a), the length of the irradiated region has a significant effect on the photocurrent. In quantum wires with a shorter arc, the current as a function of the photon energy is characterized by a single peak at $\hbar \omega=\mu$. By increasing the arc angle $\varphi$ additional peaks appear and the peak at $\hbar \omega=\mu$ decreases. We found that the position of these additional peaks is determined only by the arc length (at a fixed $R$ ) and does not depend on the radiation intensity. Figure 2(b) demonstrates that the current at $\hbar \omega=\mu$ is stronger in the wires with larger $R$ and its maximum shifts to smaller $\varphi$ with increase of $R$.

The effects of the radiation intensity and finite temperature on the photocurrent are shown in Fig. 3. In Fig. 3(a), we see that the energy dependence of the photocurrent scales with the radiation intensity without changing considerably in shape. The finite temperature [Fig. 3(b)] smoothes the peak at $\hbar \omega=\mu$ and shifts it to a lower energy. However, this effect becomes significant only at $T \sim 10 \mathrm{~K}$, implying that very 
strict temperature requirements are not needed in the experiment.

We note that in a real experiment, the situation can be more complex; however, we believe that our theory gives a right estimate for a single-mode transport. It is straightforward to modify our results in the case of wider quantum wires, where several transverse subbands are occupied. In this case, it is reasonable to assume that the circularly polarized radiation does not mix different transverse subbands. The electrons in these subbands can be considered independently and the total current is determined by contributions from all occupied subbands. Therefore, one can expect that instead of a single peak at the Fermi energy, the current dependence on the radiation frequency will show a set of equally spaced peaks (for the case of the harmonical confinement) corresponding to different transverse subbands.

The spin-independent effective mass Hamiltonian (1) is a good approximation for quantum wires without (or with a weak) spin-orbit interaction, not subjected to a static magnetic field, and with negligible effective magnetic fields due to polarized nuclear spins or magnetic impurities. In all other cases, additional terms should be included into the Hamiltonian. In fact, the ballistic transport through straight quantum wires and quantum point contacts was studied recently including some of these effects. ${ }^{26-29}$ Another known source of spin splitting in semiconductors is strain. ${ }^{30}$ Basically, we propose to directly grow quantum wires of the shape shown in Fig. 1. The curved quantum wires obtained by this method will be unstrained. Strain would be present in a bent carbon nanotube, but spin-orbit effects are expected to be small in this system. Furthermore, given the small ratio of the nanotube radius over the radius of curvature of the bending, this strain is likely to be very small. The evaluation of the importance of this effect for photocurrent generation is out of the scope of this paper.

In summary, we have demonstrated that circularly polarized electromagnetic radiation induces a current in curved ballistic quantum wires (photovoltaic effect). The current was calculated as a function of the photon energy and length of the bent segment. We have investigated the temperature and intensity dependence of the photocurrent. We found that for a realistic set of parameters, a current of the order of $10 \mathrm{pA}$ can be observed. In curved quantum wires with a short curved segment, the current dependence on photon energy shows a single peak at the Fermi energy. Larger segments give rise to additional peaks due to the wave reflection and transmission at different region boundaries.

We thank M. Dykman and D. Tománek for many fruitful discussions. This research was supported by the National Science Foundation, Grant No. NSF DMR-0312491.
*Electronic address: pershin@pa.msu.edu

${ }^{1}$ F. Hekking and Yu. V. Nazarov, Phys. Rev. B 44, 11506 (1991).

${ }^{2}$ S. Feng and Q. Hu, Phys. Rev. B 48, 5354 (1993).

${ }^{3}$ L. Fedichkin, V. Ryzhii, and V. Vyurkov, J. Phys.: Condens. Matter 5, 6091 (1993).

${ }^{4}$ A. Grincwajg, L. Y. Gorelik, V. Z. Kleiner, and R. I. Shekhter, Phys. Rev. B 52, 12168 (1995).

${ }^{5}$ F. A. Maaø and L. Y. Gorelik, Phys. Rev. B 53, 15885 (1996).

${ }^{6}$ C. S. Tang and C. S. Chu, Phys. Rev. B 53, 4838 (1996).

${ }^{7}$ S. Blom, L. Y. Gorelik, M. Jonson, R. I. Shekhter, A. G. Scherbakov, E. N. Bogachek, and U. Landman, Phys. Rev. B 58, 16305 (1998).

${ }^{8}$ Y. Levinson and P. Wölfle, Phys. Rev. Lett. 83, 1399 (1999).

${ }^{9}$ C. Niu and D. L. Lin, Phys. Rev. B 62, 4578 (2000).

${ }^{10}$ S. Blom and L. Y. Gorelik, Phys. Rev. B 64, 045320 (2001).

${ }^{11}$ G. Platero and R. Aguado, Phys. Rep. 395, 1 (2004).

${ }^{12}$ N. G. Galkin, V. A. Margulis, and A. V. Shorokhov, Phys. Rev. B 69, 113312 (2004).

${ }^{13}$ Yu. V. Pershin and C. Piermarocchi, Appl. Phys. Lett. 86, 212107 (2005).

${ }^{14}$ A. Fedorov, Yu. V. Pershin, and C. Piermarocchi, cond-mat/ 0507461, Phys. Rev. B (to be published).

${ }^{15}$ L. I. Magarill and M. V. Éntin, JETP Lett. 78, 213 (2003).

${ }^{16}$ L. I. Magarill and A. V. Chaplik, Pis'ma Zh. Eksp. Teor. Fiz. 70, 607 (1999) [JETP Lett. 70, 615 (1999)].
${ }^{17}$ Yu. V. Pershin and C. Piermarocchi, cond-mat/0502001, Phys. Rev. B (to be published).

${ }^{18}$ H. Kosaka, D. S. Rao, H. D. Robinson, P. Bandaru, T. Sakamoto, and E. Yablonovitch, Phys. Rev. B 65, 201307(R) (2002).

${ }^{19}$ E. Kapon, D. M. Hwang, and R. Bhat, Phys. Rev. Lett. 63, 430 (1989).

${ }^{20}$ E. J. Mele, P. Král, and D. Tománek, Phys. Rev. B 61, 7669 (2000).

${ }^{21}$ F. A. Maaø and Y. Galperin, Phys. Rev. B 56, 4028 (1997).

${ }^{22}$ S. Datta and M. P. Anantram, Phys. Rev. B 45, R13761 (1992).

${ }^{23}$ R. Landauer, IBM J. Res. Dev. 1, 223 (1957); M. Buttiker, Phys. Rev. Lett. 57, 1761 (1986).

${ }^{24}$ R. C. T. da Costa, Phys. Rev. A 23, 1982 (1981).

${ }^{25}$ S. N. Shevchenko and Yu. A. Kolesnichenko, JETP 92, 811 (2001).

${ }^{26}$ A. V. Moroz and C. H. W. Barnes, Phys. Rev. B 60, 14272 (1999).

${ }^{27}$ A. V. Moroz, K. V. Samokhin, and C. H. W. Barnes, Phys. Rev. B 62, 16900 (2000).

${ }^{28}$ Y. V. Pershin, J. A. Nesteroff, and V. Privman, Phys. Rev. B 69, 121306(R) (2004).

${ }^{29}$ J. A. Nesteroff, Yu. V. Pershin, and V. Privman, Phys. Rev. Lett. 93, 126601 (2004).

${ }^{30}$ Y. Kato, R. C. Myers, A. C. Gossard, and D. D. Awschalom, Nature (London) 427, 50 (2004). 